\title{
Gravitational and inertial effects of mass on the perceived heaviness of objects
}

\author{
KERRY L. GREER \\ University College, London, England
}

(Susan Henley, Sponsor)

\begin{abstract}
Previous research has drawn a distinction between mass perception and weight perception. This distinction is based on data showing that subjects' ability to judge the heaviness of an object is less acute under conditions of zero gravity. Following a discussion about the mass of an object and the gravitational and inertial effects of that mass is a proposal that this distinction between mass and weight perception is misleading. An experiment is reported that shows that the perceived heaviness of an object is a function of the magnitude of the external forces acting on that object rather than a function of the type of force, inertial or gravitational, acting on that object.
\end{abstract}

Several recent papers have purported to show that subjects' perceptions of differences in the mass of objects are less acute than their perceptions of differences in the weights of those objects (Crawford \& Kama, 1961; Rees \& Copeland, 1960; Ross \& Brodie, 1987). As Ross and Brodie pointed out, the perception of mass, without weight, can be studied on earth if the masses are supported against gravitational force and moved in the horizontal plane. Typically, subjects are required to judge weight by moving masses up and down in the vertical plane in a procedure used to determine weight perception, and to judge mass by moving the masses in the horizontal plane in a procedure used to determine mass perception. The subjective sensations that correspond to mass and weight are called heaviness (Ross \& Brodie, 1978).

Results have shown that the Weber fractions for mass are significantly higher than the Weber fractions for weight. Although these psychophysical data add importantly to the body of knowledge about human perception, Ross and Brodie's (1987) notion that the perception of the inertial effects of mass is in some way qualitatively different from the perception of the gravitational effects of mass is questionable. This notion is exemplified by such statements as: "It is interesting to consider why an object feels lighter when judged by its mass than by its weight" (p. 79), and 'In practise, 'weight' perception typically involves 'mass' perception"' (p. 78) (Ross \& Brodie, 1987). Given that physicists have known for centuries that gravitational mass and inertial mass are in fact identical, Ross and Brodie's distinction is, to say the least, surprising. It also introduces unnecessary confusion both to the description of what the subject is doing and to the

I thank Susan Henley and Nigel Harvey for their criticisms of an earlier draft of this paper, R. J. A. Audley for his helpful discussions, and Jim Chambers for making the apparatus. Address correspondence to Kerry $L$. Greer, Department of Psychology, University College, Gower Street, London WC1 6B7, England. analysis of the mechanisms responsible for judgments of heaviness. This paper aims to correct what seem to be misconceptions about the mass of an object and the inertial and gravitational effects of that mass.

To clarify this matter, it is necessary to define the terms weight, mass, and inertia. Mass is the quantity of matter that a body contains. Weight is a force that increases or decreases in proportion to like changes in mass. When a force is applied to a mass, it causes the mass to accelerate in the direction of the applied force. Weight, by means of gravity, causes the mass to be accelerated in the direction of the earth. If air resistance is neglected, objects of any mass are accelerated in the same manner. This acceleration of mass is summarized by the equation force $=$ mass $\times$ acceleration. When motion is the result of gravitational force, mass is correctly referred to as gravitational mass. Inertia is the property of an object that causes the object to resist changes in its condition or to oppose applied forces. Inertia and mass are closely related in that inertia is proportional to mass. Massive objects are less reactive to applied forces than are smaller objects. Thus, the force required to overcome the inertia of a large mass is greater than the force required to overcome the inertia of a small mass, and any resulting motion can be described by the same equation as that describing acceleration of objects under gravity: force $=$ mass $x$ acceleration. When motion is the result of forces other than gravity, mass is correctly referred to as inertial mass.

In any situation in which an object is moved by forces other than gravity, such as in the mass-perception procedure, or is moved in addition to gravity, such as in the weight-perception procedure (Ross \& Brodie, 1987), the inertial effects of mass are in operation. The resultant force can be calculated by vector addition of the component forces. The point being made here is that the difference between the weight and mass conditions of Ross and Brodie (1987) may be quantitative rather than qualitative. The purpose of this experiment was to demonstrate what 
to some might seem intuitively obvious: namely, if the inertial effects of mass and the gravitational effects of mass are equated in the objective domain of external force, the result will be corresponding equivalence in the subjective domain of perceived heaviness. To put it simply, and in the terminology of Ross and Brodie (1987), subjects' mass perception will be as good as their weight perception.

\section{METHOD}

\section{Subjects}

Six right-handed psychology students, 4 male and 2 female, were paid to participate in the experiment.

\section{Apparatus}

The stimuli consisted of two sets of 11 identical plastic containers filled with specified amounts of lead shot. Each set had a standard stimulus of $400 \mathrm{~g}$ and 10 comparison stimuli that ranged from 408 to $480 \mathrm{~g}$ in $8-\mathrm{g}$ increments. The bottles were coded for identification. The standard and comparison stimuli were slotted manually into one of two identical arms. Each arm was constructed of balsa wood $50 \mathrm{~mm}$ wide and $800 \mathrm{~mm}$ long, and weighed $90 \mathrm{~g}$. At one end of the arm, twin ball bearings (Picador) gave a low-friction bearing. Through the center of the bearing ran a rod that was gripped by a heavy-duty lockable ball joint. This ball joint enabled the balsa wood tube to swing horizontally or vertically according to its setting. A spirit level was used to ensure that the arm was properly oriented. At the end of the arm away from the bearing were two sockets at $90^{\circ}$ angles to each other. These sockets provided a snug fit for the stimuli for both vertical and horizontal directions of movement. Also at this end was a cord attachment point. In the horizontal plane, the arm plus its designated load could be given a constant horizontal pull approximately equal to that of gravity by the simple expedient of passing a cord over a ball-bearing pulley and attaching the correct stimulus. Thus, the arms could operate in three conditions of external force: vertical force (gravity) and two horizontal forces, one of which approximated gravity and the other of which was minimal. The two arms were mounted side by side on heavy stands, with the center line $1 \mathrm{~m}$ from the floor. The subject sat on a lab stool at a height of $660 \mathrm{~mm}$ and was positioned so that he/she was equidistant from both arms.

\section{Procedure}

The subject sat on a stool opposite the experimental table with eyes closed and wearing headphones through which white noise was played to mask any possible auditory cues. Using the right hand, the subject moved the stimulus-bearing arms consecutively and made verbal responses of "first" if the first arm was judged to be heavier than the second arm and "second" if the second arm was judged to be heavier than the first arm. Each subject performed under three conditions, the order of condition being counterbalanced across subjects. In Condition 1, the subject gripped the load-bearing arm between the thumb and forefinger and moved the arm rapidly up and down in the vertical plane three times. In Conditions 2 and 3, the subject again held the arm between the thumb and forefinger, but moved the arm backward and forward three times in the horizontal plane. In Condition 2 this horizontal movement was made against a constant externally imposed horizontal force approximately equal to that of gravity. In Condition 3, this horizontal force was not imposed. Each subject chose his/her own amplitude of movement, and was instructed to maintain this amplitude throughout the experiment. Most subjects chose an amplitude of approximately $160 \mathrm{~mm}$. In the terminology of Ross and Brodie (1987), Condition 1 represented the weight procedure, and Conditons 2 and 3 were both mass procedures. A BBC microcomputer was programmed to generate a random double-staircase stimulus-sequencing procedure (Cornsweet, 1962). There were 48 trials per condition. The position of the standard stimulus was randomized over all trials such that for each staircase, the standard stimulus was placed equally often on the first and second arms. The increment was $8 \mathrm{~g}$, and the response criterion was at the $50 \%$ level. The experimenter loaded the stimuli onto the specified arms and en- tered the subjects' responses into the BBC. For each trial, the staircase, the order of standard stimulus presentation, the comparison stimulus presented, and the subject's response for each trial were stored on disk.

\section{RESULTS}

The difference limens (DLs) for each subject in each condition were converted to Weber fractions using the formula $2 \Delta W /\left(W_{1}+W_{2}\right)$, where $\Delta W$ is the $\mathrm{DL}, W_{1}$ is the standard stimulus, and $W_{2}=\Delta W+W_{1}$. These fractions are presented in Table 1 .

A one-way repeated measures analysis of variance showed a significant effect of condition $[F(2,10)=43.2$, $p<.01]$. The Newman-Keul's procedure for multiple comparisons among treatment means revealed a significant difference between Conditions 1 and 3 and between Conditions 2 and 3 at the .01 level, but revealed no difference between Condition 1 and 2 . Thus, the Weber fraction for mass varied as a function of the resultant force, being the same as that for weight when the judgments were made under a constant horizontal external force equal to that of gravity but significantly greater when the judgments were made under minimal force conditions.

\section{DISCUSSION}

The results of the present experiment show clearly that in a situation in which subjective sensations of heaviness are based on objects moving through space and time, those sensations are a function of the external forces operating on the objects. It does not matter whether that force is gravitational or inertial. What does matter is its magnitude. Subjects do not perceive mass or weight, but rather the gravitational and inertial effects of mass, both of which can be described in terms of the same equation relating force and acceleration. In the weight condition (Condition 1), the stimuli were subject to a constant external force, operating in a vertical direction, known as gravity. The subjects made judgments of heaviness by moving the stimuli in the same plane as that of gravity. In one of the mass conditions (Condition 2), the subjects made judgments of heaviness by moving the stimuli in a horizontal direction against a constant external force, equal in magnitude to that of gravity, which operated in the horizontal plane. In the other mass condition (Condition 3), the subjects made heaviness judgments by moving the stimuli against minimal horizontal force. The Weber fraction for Condition 3 was significantly greater than that for Condition 2 . In other words, the subjects' mass perception varied as a function of the amount of external horizontal force acting on the object. There was no significant difference in the Weber fraction for weight and mass as measured under Condition 2 , in which the amount of external force operating was the same; the Weber fraction for mass as measured under Condition 3, however, was significantly greater than the Weber fraction for weight.

Ross and Brodie (1987) considered the question of why an object should feel lighter when judged by its mass rather than by its weight. In light of the present results, it seems to be more accurate and far more infor-

Table 1

Weber Fractions for Each Subject in Each Condition

\begin{tabular}{cccc}
\hline Subject & Condition 1 & Condition 2 & Condition 3 \\
\hline 1 & 0.058 & 0.077 & 0.182 \\
2 & 0.039 & 0.058 & 0.131 \\
3 & 0.058 & 0.058 & 0.113 \\
4 & 0.039 & 0.039 & 0.077 \\
5 & 0.039 & 0.039 & 0.113 \\
6 & 0.039 & 0.039 & 0.131 \\
$M$ & 0.045 & 0.051 & 0.125 \\
\hline
\end{tabular}


mative to consider why an object feels lighter when the magnitude of external force acting on that object is greatly reduced. This experiment does not address this question directly. It does, however, have some bearing on previous explanations of the psychophysical data. Rees and Copeland (1960) and Crawford and Kama (1961) proposed that sensations of heaviness were a function of the amount of force used by the subject to move the object. Ross and Brodie (1987) suggested that "the reduction in apparent heaviness for mass (without weight) is more likely to be due to the loss of the reactive force contributed by the weight of an object than to a reduction in applied force"' (p. 80). The data obtained in the present experiment are consistent with both these views. The subjects did have to apply approximately the same force to overcome the resistance to movement in Conditions 1 and 2, but far less force to overcome resistance to movement in Condition 3. Common sense tells us that we can apply more force than is necessary to lift or move objects, but much information is given by the interaction between applied force, resistance to movement, and acceleration of objects. It is precisely this information that is degraded in Condition 3 of this experiment and in the mass conditions of previous work. It would be a relatively simple matter to investigate this relationship directly.

In conclusion, the results of this experiment do not support the idea that mass perception is based on mechanisms that differ from those that underlie weight perception. One cannot rule out the possibility that subjects' perceptions of the inertial effects of mass are based on the same mechanism as that underlying subjects' perceptions of gravitational effects of mass. The data presented here are consistent with the view that the perceived heaviness of an object is a function of the magnitude of the resolved forces operating on that object.

\section{REFERENCES}

Cornsweet, T. N. (1962). The staircase-method in psychophysics. American Journal of Psychology, 75, 485-491.

Crawford, S. M., \& KAMA, W. N. (1961). Remote handling of mass (Report No. ASD TR 61-627). Wright-Patterson Air Force Base, Ohio.

ReEs, D. W., \& Copeland, N. K. (1960). Discrimination of differences in mass of weightless objects (WADD Tech. Report No. 60601). Wright-Patterson Air Force Base, Ohio.

Ross, H. E., \& BRODIE, E. (1987). Weber fractions for weight and mass as a function of stimulus intensity. Quarterly Journal of Experimental Psychology, 39A, 77-88.

(Manuscript received March 23, 1988.) 